\title{
Remèdes actifs et passifs
}

\section{Enseignements}

\author{
Président : C. Bocquillon* \\ Rapporteur : E. Tormos** \\ * Université de Montpellier \\ ** CNR, Lyon
}

La crue d'un cours d'eau est un événement qui se manifeste, comme chacun le sait, par des débordements plus ou moins importants, pouvant alors occasionner des dégâts matériels mais aussi des pertes humaines.

Pourquoi ces pertes et dégâts ? Parce que de tout temps, l'Homme s'est rapproché de l'eau, y trouvant :

- Des ressources pour vivre ;

- Une voie de communication ;

- Plus récemment, l'Homme y a trouvé une force motrice pour produire de l'électricité, source d'énergie nécessaire à l'industrie :

- Plus récemment encore, comme le dit M. Mottet dans un article paru dans la Revue de Géographie de Lyon, l'exode rural a peuplé le fond des vallées susceptibles de fournir un emploi dans l'industrie ou des terres planes adaptées à l'agriculture mécanisée :

- Enfin, actuellement, certaines vallées ont retrouvé une activité résultant d'un développement touristique. En effet, le touriste veut le soleil, des activités sportives ou culturelles et de l'Eau. Ainsi, un camping à $5 \mathrm{~km}$ de la rivière ne l'intéresse pas, mais il trouve de multiples avantages à installer sa caravane dans celui qui est près de l'eau, c'est-à-dire dans le lit majeur.

Dès lors, les sinistrés ou ceux qui risquent de l'être en fonction des événements catastrophiques produits par ailleurs, demandent à être protégés. Serait-il alors illégitime que cela soit fait ?

Aussi, les Responsables à tous niveaux ont-ils à engager une réflexion profonde sur les mesures susceptibles d'atténuer voire supprimer les effets de ce type d'événements en matière de prévention.

C'était l'objet de la question III des $23^{\mathrm{e}}$ journées de l'hydraulique de la S.H.F.
Pour traiter des remèdes des actifs et passifs, 23 rapports ont été retenus, présentés et commentés.

Trois questions principales ont été posées à l'ensemble des auteurs de Communications :

a. Pourquoi le bon hydrogramme est-il obtenu ? C'est-àdire est-ce que sa distorsion est réalisable et est supportable ailleurs?

b. Comment le sophisme de la goutte d'eau est évité pour l'aval! (entre autres termes, est-ce que le cumul d'aggravations minimes est aussi minime ?)

c. Pourquoi le projet est économiquement correct. Notamment que se passe-t-il quand on a décidé de prendre une borne administrative $\left(1 / 100^{\mathrm{c}}\right.$ pour les constructions d'habitation) si cette borne est franchie ?

Bien que la majorité des rapports corresponde à des études de cas, les conclusions suivantes peuvent être tirées :

1. Les dispositifs de protection contre les crues sont variés (barrages-réservoirs à buts multiples, endiguement, amélioration de la débitance, barrages écrêteurs...). En général, ce n'est pas un type unique qui est utilisé sur un cours d'eau mais une combinaison de plusieurs de ces dispositifs.

2. Il est nécessaire de bien connaître les modifications apportées aux lignes d'eau consécutives à une modification des surfaces inondées. Et aujourd'hui, avec les moyens informatiques disponibles, nous savons très bien calculer les écoulements en rivière et les progrès effectués ces dernières années dans l'hydro- 
morphologie fluviale nous permettent d'estimer aussi les effets d'une modification du lit ou de l'écoulement des débits. Pratiquement toutes les études présentées au cours de cette séance ont eu recours à des modèles mathématiques.

3. Il est nécessaire de veiller à ce que tout système de protections contre les crues mis en place en un secteur de la rivière n'aggrave pas la situation des riverains d'amont ou d'aval. En cela les schémas d'aménagement et gestion des rivières (SAGE) qui se mettent en place progressivement sont une très bonne disposition.

4. Le dimensionnement des dispositifs de protection doit tenir compte d'un développement économique mais aussi de l'urbanisation. Certains dégâts apparus lors des récentes crues montrent la nécessité d'une telle prise en compte.

5. Une réglementation dans l'occupation des sols est indispensable. En effet, il est quasiment impossible de supprimer totalement les inondations qui peuvent avoir d'ailleurs des impacts positifs (alimentation des nappes, irrigation).
6. Il est nécessaire et possible grâce, là aussi, à l'informatique de procéder à des études économiques qui permettront le choix du niveau de protection et les dispositions permettant de le satisfaire sans perdre de vue que certaines contraintes écologiques politiques ou tout autre, peuvent conduire à l'abandon de celles-ci.

7. Une information du public (annonce de crues, système d'alerte) doit être une mesure à prévoir à chaque fois qu'un dispositif de protection est mis en place.

8. La cohérence des systèmes de protection mis en place doit être assurée en s'intéressant en particulier aux crues extrêmes de façon à éviter de transformer un cataclysme naturel en catastrophe liée à des ruptures inopinées d'ouvrages.

Deux autres points seraient à rajouter à cette liste :

9. Nécessité de prendre en compte la structure des écosystèmes aquatiques.

10. La dynamique destructrice a, parmi les géographes, ses partisans qui considèrent que la rivière doit toujours se reconstruire, se régénérer en remaniant les sédiments de son lit. Dans ces conditions, les projets exigent tout au long de leur vie «suivi et maintenance» faute de quoi ils génèreront des risques supplémentaires.

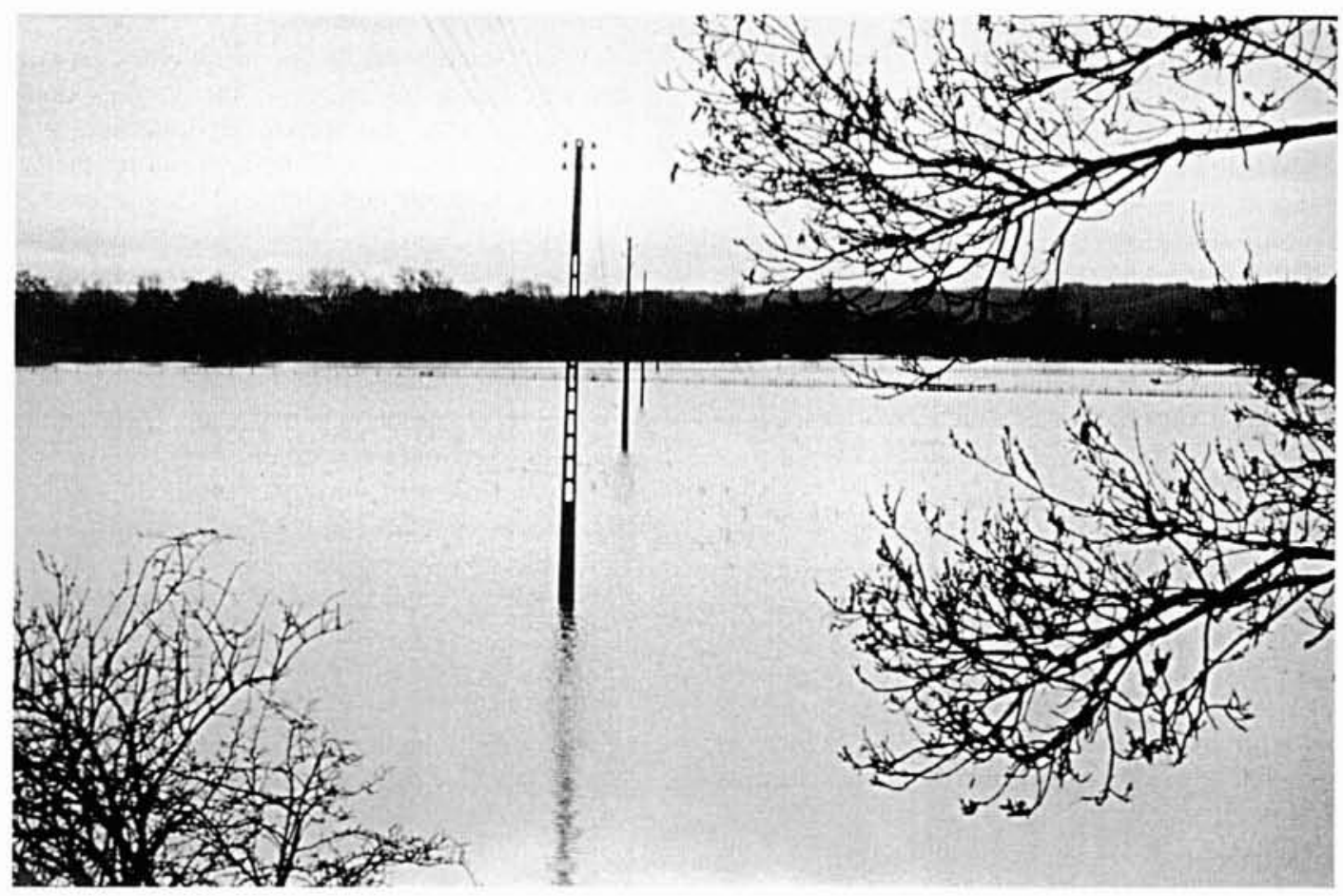

Inondations dans la région de La Flèche (photothèque EDF, photo Marc Morceau). 